\title{
Copper oxide, ionic liquid and Mn(III) Salen modified carbon paste electrode as selective electrochemical sensor for determination of droxidopa in the presence of carbidopa
}

\author{
Mohammad Reza Aflatoonian ${ }^{a, b}$, Somayeh Tajik ${ }^{c, a, *}$, Behnaz Aflatoonianc, \\ Iran Sheikh Shoaied, Mahdieh Sheikhshoaiee, Hadi Beitollahif,* \\ a Research Center for Tropical and Infectious Diseases, Kerman University of Medical Sciences, \\ Kerman, Iran \\ ${ }^{b}$ Leishmaniasis Research Center, Kerman University of Medical Sciences, Kerman, Iran \\ ${ }^{c}$ Neuroscience Research Center, Kerman University of Medical Sciences, Kerman, Iran \\ 'Department of Chemistry, Faculty of Science, Shahid Bahonar University of Kerman, Kerman \\ 76175-133, Iran
}

eDepartment of Mining Engineering, Shahid Bahonar University of Kerman, Kerman, Iran

fEnvironment Department, Institute of Science and High Technology and Environmental Sciences, Graduate University of Advanced Technology, Kerman, Iran

Received: 23 September 2019, Accepted: 15 November 2019, Published: 02 December 2019

\begin{abstract}
A novel electrochemical sensor for the selective and sensitive detection of droxidopa in presence of carbidopa at physiological $\mathrm{pH}$ was developed by the bulk modification of carbon paste electrode (CPE) with copper oxide $(\mathrm{CuO})$, ionic liquid and $\mathrm{Mn}$ (III) salen (CuO-IL-MS/CPE). Large peak separation, good sensitivity and stability allow this modified electrode to analyze droxidopa individually and in the presence of carbidopa. Applying differential pulse voltammetry (DPV), a linear dynamic range of 0.5-600.0 $\mu \mathrm{M}$ with detection limit of $0.2 \mu \mathrm{M}$ was obtained for droxidopa. Finally, the proposed method was applied to the determination of droxidopa and carbidopa in real samples.
\end{abstract}

Keywords: Droxidopa; carbidopa; $\mathrm{Mn}$ (III) salen; CuO nanoparticles; electrochemical sensor.

\section{Introduction}

L-Threo-3, 4-dihydroxyphenylserine (droxidopa) is a synthetic precursor amino acid converted both peripherally and centrally into norepinephrine by the ubiquitous enzyme, dopa-decarboxylase. Droxidopa is capable of crossing the protective blood-brain barrier while norepinephrine and epinephrine are not able to pass. Therefore, it is effective for the treatment of frozen gait and dizziness on standing associated with Parkinson's disease (PD) and for the treatment of orthostatic hypotension and also

*Corresponding authors: Somayeh Tajik, Hadi Beitollahi

Tel: +98 3426226613, Fax: +98 3426226617

Eurasian Chem. Commun., (2020) 387-397

E-mail: tajik_s1365@yahoo.com, h.beitollahi@yahoo.com 
nondiabetic autonomic neuropathy, or dopamine $\beta$ - hydroxylase deficiency. Moreover, droxidopa alone or in combination with one or more further pharmaceutically active compounds such as pain relievers (acetaminophen, flupirtine, and tramadol) is useful in the treatment of fibromyalgia [1-7].

Parkinson's disease is believed to be related to low levels of the neurotransmitter dopamine in the brain. Despite of the good results for the Parkinson's disease treatment with levodopa (LD), a set of side effects has been reported from the LD consumption, as motor and non-motor fluctuations, dyskinesias, neuropsychiatric complications and sleep disturbances. Carbidopa [(-) $\quad-\mathrm{L} \quad-2 \quad-\quad(3, \quad 4-$ dihydroxybenzyl) -2-hydrazinopropionic acid] is a catecholamines of an alkyl amine chain that is bound to a benzene ring carrying two hydroxyl groups. Aromatic-L-amino-acid decarboxylase (DOPA decarboxylase or DDC), which is a significant enzyme in biosynthesizing L-tryptophan to serotonin and L-DOPA to dopamine, is inhibited by carbidopa [8-12]. This condition leads to entrance of greater LDOPA to brain that may decline signs of Parkinson's illness. Furthermore, since DDC is one of the enzymes necessary to convert droxidopa to its active metabolite norepinephrine, suppression of peripheral DDC with the use of carbidopa had to lead to diminishing consequences of peripheral generation of norepinephrine.

In view of the above considerations, this drugs analysis has played a vital role in drug quality control. Droxidopa and carbidopa are electroactive substances with hydroxide radical, so electrochemical methods are convenient for detecting droxidopa and carbidopa. Compared with other reported analytical methods, electrochemical methods possess the advantages such as, reliability, simple operation, capability of miniaturization, being portable, fast response, high sensitivity, excellent selectivity, excellent stability, and low cost. In addition, electrochemical methods provide a practical tool for online or field monitoring [13-27]. However, a main disadvantage is that droxidopa and carbidopa are oxidized at nearly same potential with poor sensitivity at unmodified usual electrodes. The overlapping of the oxidation voltammetric peaks makes the simultaneous determination vastly difficult.

Chemically modified electrode (CME) has emerged as an efficient and versatile approach and has attracted considerable interest over the past two decades due to the advantages in terms of reduced costs, automatic and fast analysis, high sensitivity and selectivity [28-35].

After the first usage of carbon paste as the electrode material by Adams, carbon paste electrodes (CPE) have attracted highly the analysts due to its advantages such as steady response, easy renewability, and low ohmic resistance [36-40].

Recent developments in advanced nanotechnology and ininnovative chemisensors, nanomaterials, and nano devices have been regulating a key task in the fabrication and improvement of very precise, perceptive, accurate, sensitive, and consistently efficient chemical sensors. The exploration for even small electrodes accomplished in nano-level imaging and in controlling doped nanomaterials, doping agents (hostguest), biological, chemical, pathological samples, and chemical sensors has extended scientists' awareness for control monitoring, especially in the fields of environmental safety and health monitoring. 
Recently, the metal oxides, as widely distributed materials, are increasingly reported as the surface modified materials of working electrodes in electrochemical detection due to their low-cost, large BET surface area and tremendous adsorption capacity [41-45].

Copper oxide $(\mathrm{CuO})$ as a typical $\mathrm{p}$ type transition metal oxide with a narrow band gap of $1.2 \mathrm{eV}$ has been extensively studied due to its low cost, non-toxicity, and favorable pseudo capacitive characteristics. To date, several $\mathrm{CuO}$ nanostructures such as nanoflowers, nanoleaves, nanoplatelets, nanoribbons, nanorods, nanorings, nanosheets, nanotubes, nanowires, and nanowhiskers have been synthesized. It is expected that $\mathrm{CuO}$-carbon materials not only increase the electrochemical activity, but also retain the mechanical stability of $\mathrm{CuO}$ [46-49].

In this study, $\mathrm{CuO}$, ionic liquid and Mn (III) salen modified carbon paste electrode (CuO-IL-MS/CPE) was fabricated. The electrochemical behavior of CuO-IL-MS/CPE against droxidopa was assessed using cyclic voltammetry (CV), differential pulse voltammetry (DPV) and chronoamperometry. Further, $\mathrm{CuO}-\mathrm{IL}-\mathrm{MS} / \mathrm{CPE}$ was used as electrochemical sensor for simultaneous determination of droxidopa and carbidopa. The fabricated CuO-ILMS/CPE exhibits a selective and stable electrocatalytic performance for the determination of droxidopa in the presence of carbidopa in urine and water samples.

\section{Experimental}

Apparatus and chemicals

An Autolab potentiostat/galvanostat (PGSTAT 302N, Eco Chemie, the Netherlands) was applied for measuring electrochemicals. General Purpose Electrochemical System (GPES) software was employed to control conditions of experiments. A conventional three electrode cell was used at $25 \pm 1^{\circ} \mathrm{C}$. $\mathrm{An} \mathrm{Ag} / \mathrm{AgCl} / \mathrm{KCl}(3.0$ $\mathrm{M})$ electrode, a platinum wire, and $\mathrm{CuO}$ IL-MS/CPE were used as the reference, auxiliary and working electrodes, respectively; afterwards, $\mathrm{pH}$ was measured by a Metrohm $710 \mathrm{pH}$ meter.

Droxidopa, carbidopa, and all the remaining reagents had an analytical grade. They have been prepared via Merck (Darmstadt, Germany). Orthophosphoric acid and the related salts that were above the $\mathrm{pH}$ range of 2.0-9.0 were used for preparing the buffer solutions. Ionic liquid (n-hexyl-3methylimidazolium hexafluoro phosphate) was purchased from SigmaAldrich Co. CuO nanoparticles [50] and Mn (III) Salen [51] were synthesized as reported previously.

\section{Preparing electrode}

$\mathrm{CuO}-\mathrm{IL}-\mathrm{MS} / \mathrm{CPE}$ was prepared by hand mixing $0.01 \mathrm{~g}$ of MS with $0.95 \mathrm{~g}$ graphite powder and $0.4 \mathrm{~g} \mathrm{CuO}$ nanoparticles with a mortar and pestle. Then, $0.3 \mathrm{~mL}$ IL and $0.6 \mathrm{~mL}$ of paraffin oil were added to the above mixture and mixed for 15 min until a uniformly wetted paste was obtained. Then, the paste has been packaged to the bottom of a glass tube (ca. $3.4 \mathrm{~mm}$ i.d.\& $10 \mathrm{~cm}$ long). A copper wire placed over carbon paste led to an electrical contact. If necessary, a novel surface has been gained by pushing an excessive paste out of tube and polishing with a weighing paper.

For comparison, MS modified CPE electrode (MS-CPE) without $\mathrm{CuO}$ nanoparticles and ionic liquid, $\mathrm{CuO}$ carbon paste electrode (CuO/CPE) without $\mathrm{MS}$ and ionic liquid, and unmodified CPE in the absence of MS, $\mathrm{CuO}$ nanoparticles and ionic liquid were also prepared in the same way.

\section{Preparing real samples}

Samples of urine have been kept in a refrigerator directly after gathering. Ten 
millilitres of samples have been centrifuged for fifteen minutes at 2,000 $\mathrm{rpm}$. The supernatant has been filtered by a $0.45 \mu \mathrm{m}$ filter. Next, various volumes of solution has been transported into a 25 millilitres volumetric flask and diluted to the mark with PBS $(\mathrm{pH}=7.0)$. This diluted urine samples were anaesthetized with different amounts of droxidopa and carbidopa. Content of droxidopa and carbidopa has been analyzed by the suggested procedure by employing the standard addition method.

Tap water (Kerman drinking water, Kerman, Iran), was filtered to remove all of the suspended particulate matter. This diluted water sample was anaesthetized with different amounts of droxidopa and carbidopa. Content of droxidopa and carbidop has been analyzed by the suggested procedure employing the standard addition method.

\section{Results and discussion}

Morphological study

Scanning electron microscopy has been used to examine the product morphology (Figure 1). As can be seen, $\mathrm{CuO}$ nanoparticles were synthesized in nanometer sizes.

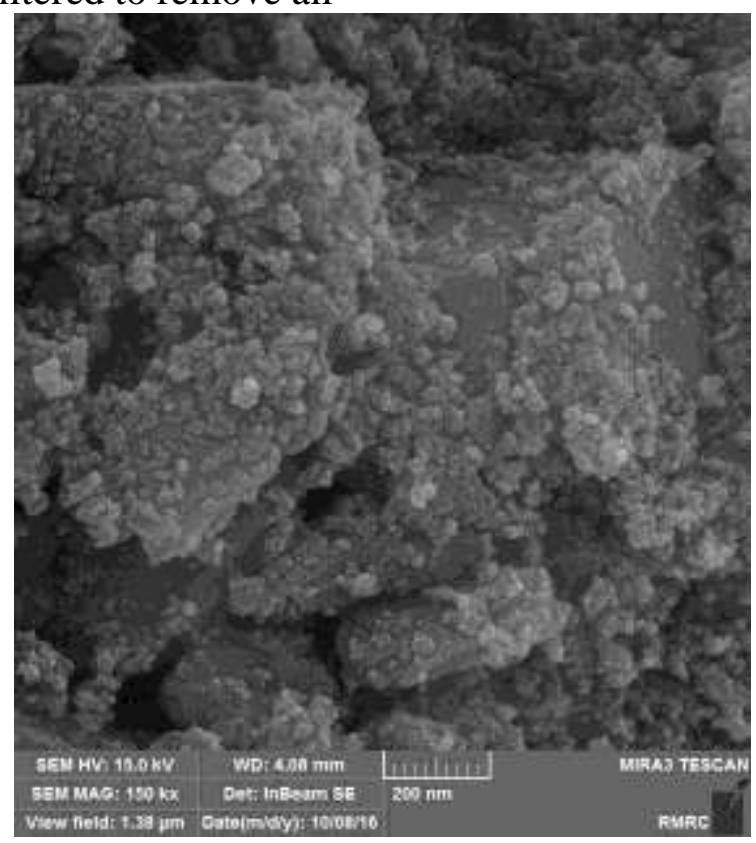

Figure 1. SEM image of $\mathrm{CuO}$ nanoparticles.

Electrochemical properties of droxidopa on $\mathrm{CuO}-\mathrm{IL}-\mathrm{MS} / \mathrm{CPE}$ surface

The electrochemical behaviour of droxidopa was dependent on the $\mathrm{pH}$ value of the aqueous solution (Figure 2A). Therefore, $\mathrm{pH}$ optimization of the solution seems to be necessary in order to obtain the electrocatalytic oxidation of droxidopa. Thus, the electrochemical behaviour of droxidopa was studied in $0.1 \mathrm{M}$ PBS in different $\mathrm{pH}$ values $(2.0<$
$\mathrm{pH}<9.0$ ) at the surface of $\mathrm{CuO}$-ILMS/CPE by CV. It was found that the electrocatalytic oxidation of droxidopa at the surface of CuO-IL-MS/CPE was more favoured under neutral conditions than in acidic or basic medium. This appears as a gradual growth in the anodic peak current. Thus, the $\mathrm{pH} 7.0$ was chosen as the optimum $\mathrm{pH}$ for electrocatalysis of droxidopa oxidation at the surface of $\mathrm{CuO}-\mathrm{IL}-\mathrm{MS} / \mathrm{CPE}$. 


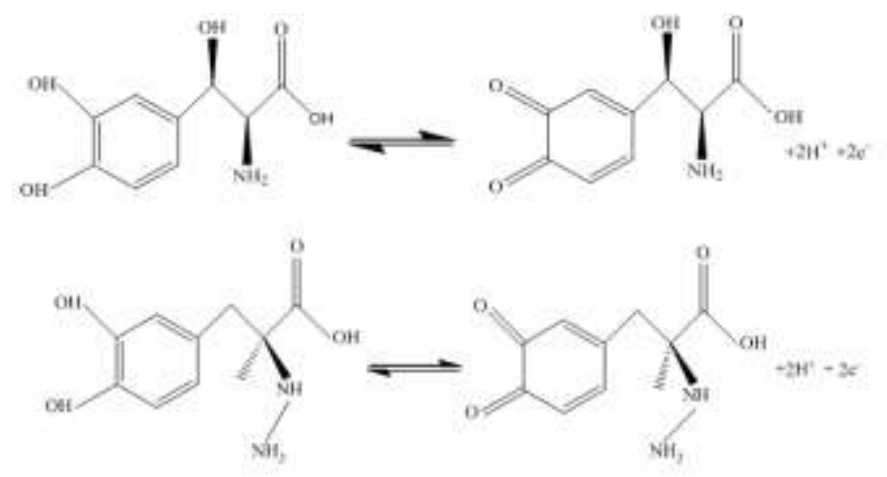

(A)

(B)

Figure 2. Electrochemical mechanisms for oxidation of droxidopa (A) and carbidopa (B).

Also, the electrochemical behaviour of carbidopa was dependent on the $\mathrm{pH}$ value of the aqueous solution (Figure 2B). Results showed that $\mathrm{pH} 7.0$ was the optimum $\mathrm{pH}$ for electrocatalysis of carbidopa oxidation at the surface of
CuO-IL-MS/CPE. Figure 3 depicts the $\mathrm{CV}$ responses for the electrochemical oxidation of $100.0 \mu \mathrm{M}$ droxidopa at unmodified $\mathrm{CPE}$ (curve b), $\mathrm{CuO} / \mathrm{CPE}$ (curve d), MS/CPE (curve e) and $\mathrm{CuO}$ IL-MS/CPE(curve f).

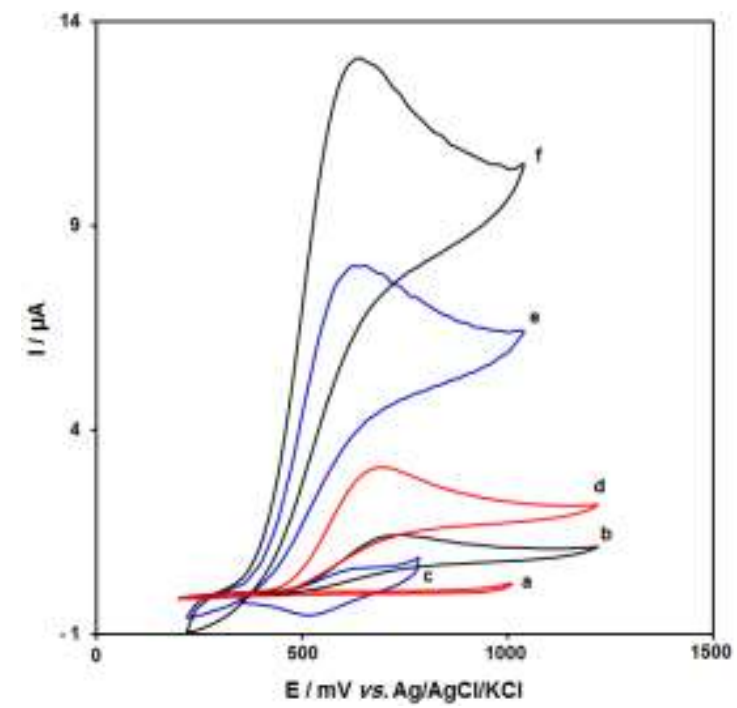

Figure 3. CVs of (a) unmodified CPE in 0.1 MPBS (pH 7.0); (b) as (a) unmodified CPE in 100.0 $\mu \mathrm{M}$ droxidopa; (c) as (a) at the surface of CuO-IL-MS/CPE; (d) as (b) at the surface of CuO/CPE; (e) as (b) at the surface of MS/CPE; (f) as (b) at the surface of CuO-IL-MS/CPE. In all cases the scan rate was 10 $\mathrm{mV} \mathrm{s}^{-1}$.

Figure 3 shows that the anodic peak potential is about $720 \mathrm{mV}$ for droxidopa oxidation on the bare CPE surface (curve b) and $640 \mathrm{mV}$ on the CuO-IL-MS/CPE surface (curve f). According to these curves, the peak potential obtained for the oxidation of droxidopa on the modified electrode surface switches about $80 \mathrm{mV}$ to negative values than that on the bare electrode surface. Based on the droxidopa oxidation on the MS/CPE (curve e) and CuO-IL-MS/CPE (curve f) surfaces, the anodic peak current has been increased on the CuO-IL-MS/CPE compared to the MS/CPE, suggesting the enhancement of the peak currents by ionic liquids (ILs) and $\mathrm{CuO}$ nanoparticles presence in the CPE. A great improvement of $\mathrm{CuO}$ on IL surface was seen on the electrochemical response, probably owing to potent properties of $\mathrm{CuO}$, including great surface area, strong chemical stability and proper electrical conductivity. 
Effect of scan rate on the results

Researchers investigated the impact of the rates of potential scan on droxidopa oxidation current (Figure 4). Findings indicated induction of enhancement in the current of the peak by the increased potential scan rate. Additionally, diffusion in oxidation processes are monitored, as inferred by the linear

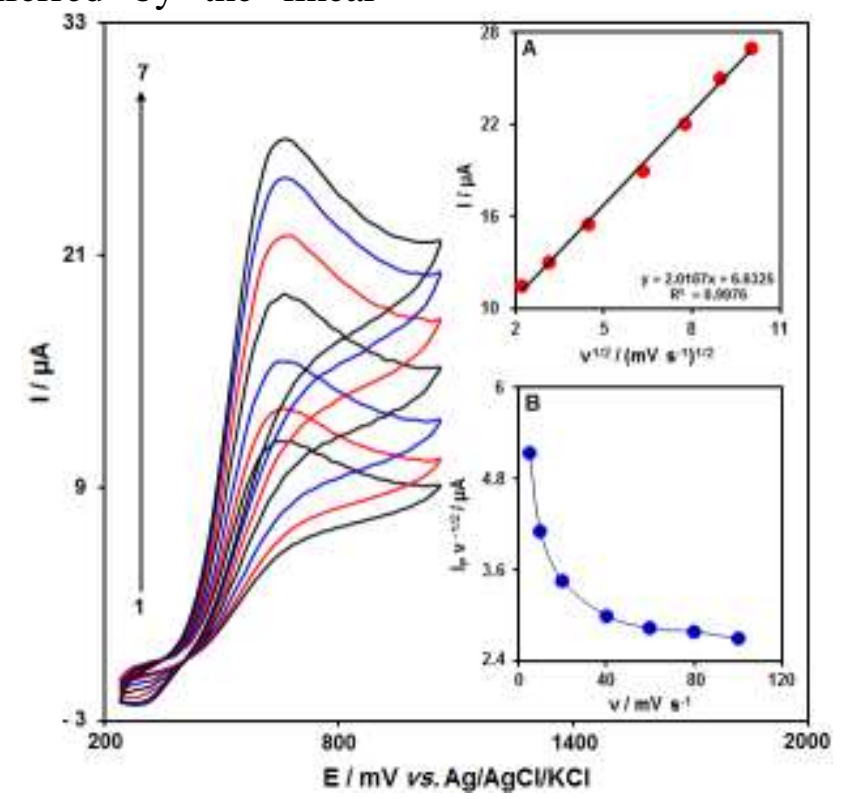

Figure 4. $\mathrm{CV}$ s of $\mathrm{CuO}-\mathrm{IL}-\mathrm{MS} / \mathrm{CPE}$ in $0.1 \mathrm{M}$ PBS ( $\mathrm{pH}$ 7.0) containing $100.0 \mu \mathrm{M}$ of droxidopaat various scan rates; numbers 1-7 correspondto 5, 10, 20, 40, 60, 80 and $100 \mathrm{mV} \mathrm{s}^{-1}$, respectively. Insets: (A) variation of anodic peak current with square root of scan rate and (B) variation of scan rate-normalized current $\left(\mathrm{Ip} / \mathrm{v}^{1 / 2}\right)$ with scan rate.

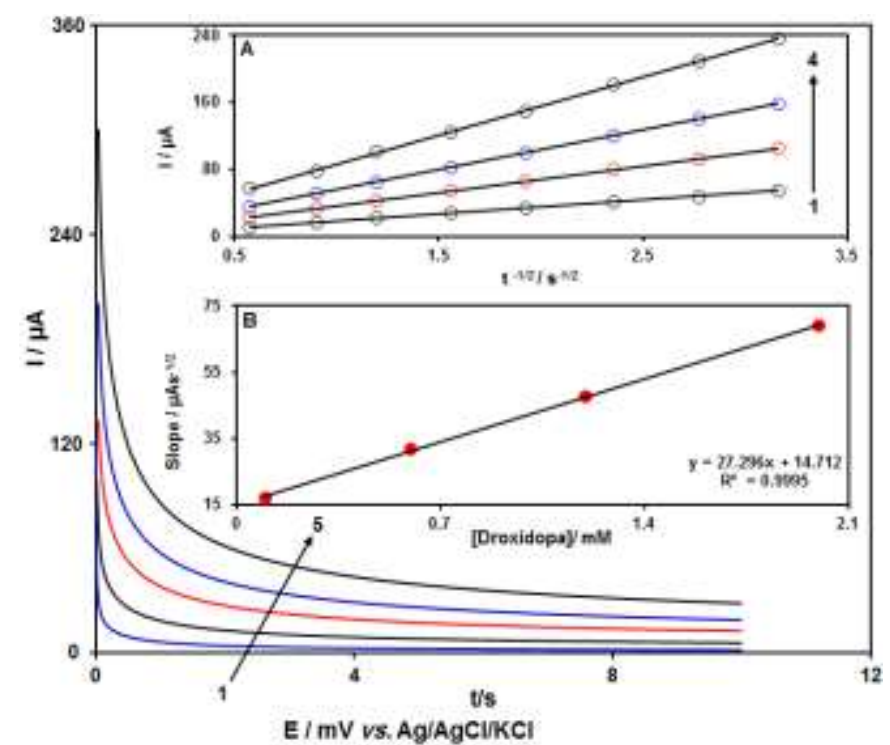

Figure 5. Chronoamperograms obtained at CuO-IL-MS/CPEin 0.1 M PBS (pH 7.0) for different concentrations of droxidopa. The numbers 1-5 correspond to $0,0.1,0.6,1.2$ and $2.0 \mathrm{mM}$ of droxidopa. Insets: (A) Plots of I vs. $\mathrm{t}^{-1 / 2}$ obtained from chronoamperograms 2-5. (B) Plot of the slope of the straight lines against droxidopa concentrations. 
Chronoamperometric analyse

Chronoamperometric measurements of droxidopa at CuO-IL-MS/CPE were conducted by adjusting the working electrode potential at $0.7 \mathrm{~V}$ versus $\mathrm{Ag} / \mathrm{AgCl} / \mathrm{KCl}(3.0 \mathrm{M})$ for different concentrations of droxidopa (Figure 5) in PBS (pH 7.0). For electroactive materials (droxidopa in this case) with a diffusion coefficient of $\mathrm{D}$, the Cottrell equation describes current seen for electrochemical reaction at the mass transport limited condition:

$$
\mathrm{I}=\mathrm{nFAD}{ }^{1 / 2} \mathrm{C}_{\mathrm{b}} \pi^{-1 / 2} \mathrm{t}^{-1 / 2}
$$

where $\mathrm{D}$ and $\mathrm{C}_{\mathrm{b}}$, respectively, represent diffusion coefficient $\left(\mathrm{cm}^{2} \mathrm{~s}^{-1}\right)$ and bulk concentration $\left.(\mathrm{mol} \mathrm{cm})^{-3}\right)$. Experimental plots of I versus $t^{-1 / 2}$ were used with the best fits for various concentrations of droxidopa (Figure 5A). Then, the resultant straight lines slopes were drawn against droxidopa concentrations (Figure 5B). According to the resultant slope and the Cottrell equation, mean values of $\mathrm{D}$ were $7.7 \times 10^{-6} \mathrm{~cm}^{2} / \mathrm{s}$ for droxidopa.

\section{Calibration curve and $L O D$}

The electro-oxidation peak currents of droxidopa at CuO-IL-MS/CPE surface can be applied to define droxidopa in the solution. Since the increased sensitivity and more suitable properties for analytical utilizations are considered as the benefits of differential pulse voltammetry (DPV), CuO-IL-MS/CPE in $0.1 \mathrm{M}$ PBS consisting of different distinct concentrations of droxidopa was used to conduct DPV experiments (Figure 6). It was found that the electrocatalytic peak currents of droxidopa oxidation at CuO-IL-MS/CPE surface linearly depended on droxidopa concentrations above the range of 0.5 $600.0 \mu \mathrm{M}$ (with a correlation coefficient of 0.9998), while determination limit $(3 \sigma)$ was achieved to be $0.2 \mu \mathrm{M}$.

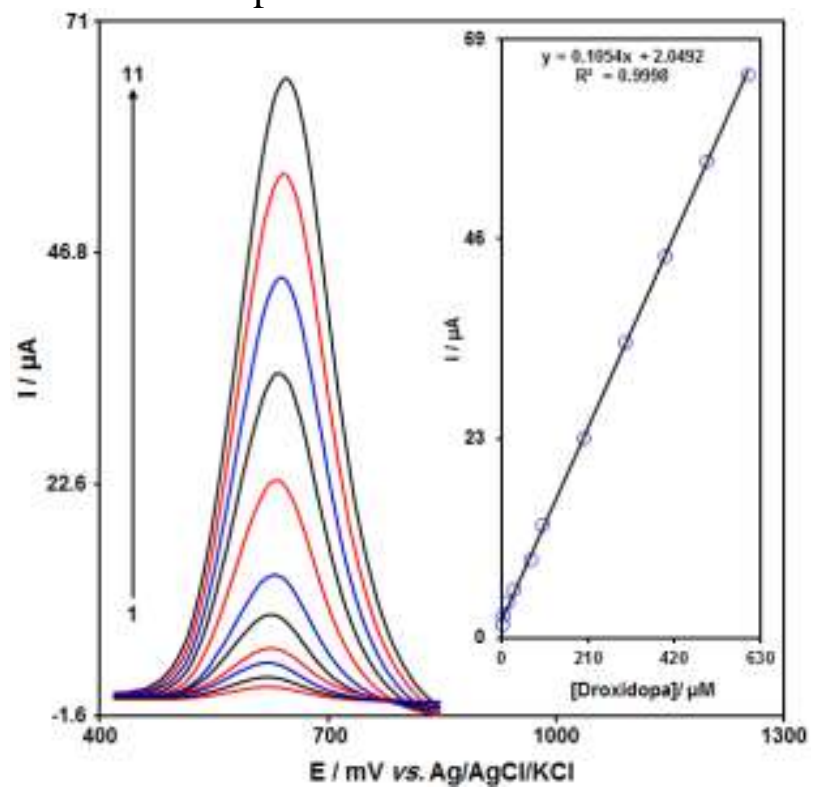

Figure 6. DPVs of CuO-IL-MS/CPEin 0.1 M PBS (pH 7.0) containing different concentrations of droxidopa Numbers 1-11 correspond to 0.5, 5.0, 15.0, 30.0, 70.0, 100.0, 200.0, 300.0, 400., 500.0 and $600.0 \mu \mathrm{M}$ ofdroxidopa. The inset shows the plot of the peak current as a function of the droxidopa concentration in the range of $0.5-600.0 \mu \mathrm{M}$.

Determination of droxidopa in the presence of carbidopa

We have not seen any report about using a CPE modified with CuO-IL-MS for determining droxidopa and carbidopa. Moreover, due to the reality that electrochemical detection of droxidopain at the front of carbidopa with the help of un- 
modified electrodes has the caveat of interventions by carbidopa because of relative adjacent oxidation capacities of the two specimens, it can be regarded as a crucial phase. Such a phase has been conducted by simultaneous alterations of analytes concentrations and achieving
DPVs (Figure 7). Findings reported certain anodic at 620 and $800 \mathrm{mV}$ for droxidopaand carbidopa oxidation, proving the use of CuO-IL-MS/CPE electrode. These two analytes can be detected without severe interventions from each another (Figure 7).

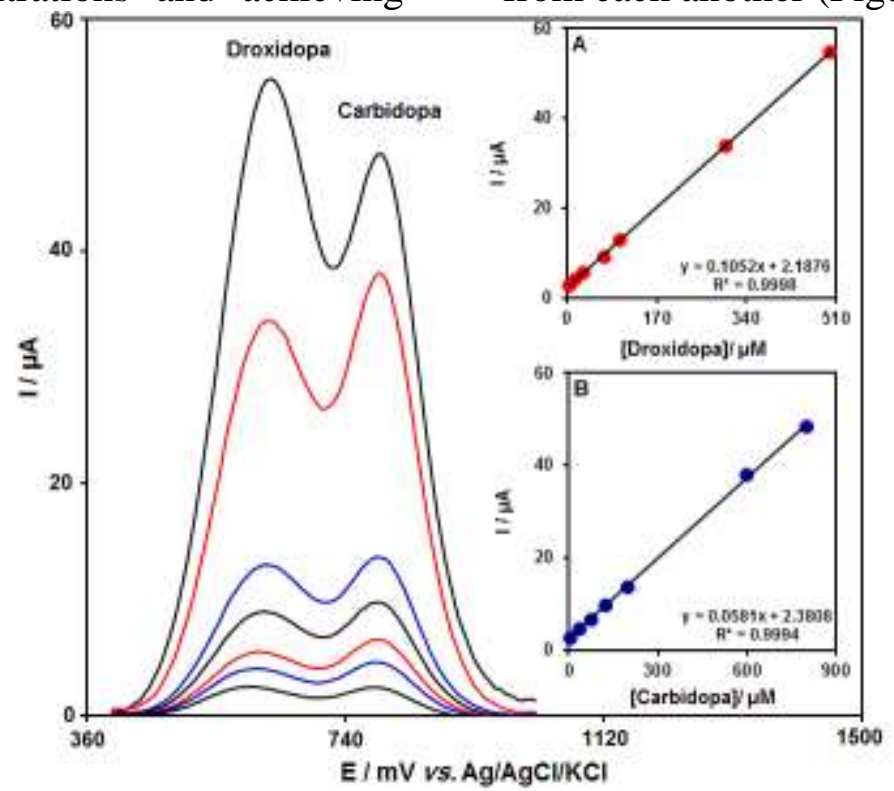

Figure 7. DPVs achieved at CuO-IL-MS/CPE surface in 0.1 M PBS (pH 7.0) consisting of various concentrations of droxidopa and carbidopa. DPVs from internal to external respectively is corresponding to $5.0+5.0,15.0+35.0,30.0+75.0,70.0+125.0,100.0+200.0,300.0+600.0$ and $500.0+800.0 \mu \mathrm{M}$ of droxidopa and carbidopa. Insets: (A) plot of Ip versus droxidopa concentration and (B) plot of Ip versus carbidopa concentration.

\section{Interference studies}

The influence of various substances as compounds potentially interfering with the determination of droxidopa was studied under optimum conditions. The potentially interfering substances were chosen from the group of substances commonly found with droxidopa in pharmaceuticals and/or in biological fluids. The tolerance limit was defined as the maximum concentration of the interfering substance that caused an error of less than $\pm 5 \%$ in the determination of droxidopa. According to the results, 1lysine, glucose, NADH, acetaminophen, uric acid, 1-asparagine, 1-serine, 1cysteine, 1-cystine, 1-threonine, 1-proline, $\mathrm{N}$-acetyl cysteine, histidine, glycine, methionine, tryptophan, glutathione, phenylalanine, lactose, saccarose, fructose, benzoic acid, methanol, ethanol, urea, $\mathrm{Ca}^{2+}, \mathrm{Mg}^{2+}, \mathrm{Al}^{3+}, \mathrm{NH}_{4}^{+}$, $\mathrm{Fe}^{2+}, \mathrm{Fe}^{3+}, \mathrm{F}-, \mathrm{SO}_{4}{ }^{2-}, \mathrm{S}^{2-}$ and uric acid did not show interference in the determination of droxidopa, but ascorbic acid, dopamine, levodopa, epinephrine and norepinephrine showed interferences. Although ascorbic acid shows interference, its interference can be minimized, if necessary, by using ascorbic oxidase enzyme, which exhibits high selectivity for the oxidation of ascorbic acid.

\section{Analysis of real samples}

To assess the applicability of the application of the modified electrode for the determination of droxidopa and carbidopa in real samples, the described method was applied to the determination 
of droxidopa and carbidopain in urine and water samples. Therefore, the standard addition technique was applied. Table 1 reports the results. Acceptable recoveries of droxidopa and carbidopa were observed, and reproducible results was shown with regard to the mean relative standard deviation (R.S.D.).

Table 1. Determination of droxidopa and carbidopa in real samples. All the concentrations are

\begin{tabular}{ccccccccc}
\hline \multicolumn{1}{c}{ Sped } & \multicolumn{2}{c}{ Found } & \multicolumn{2}{c}{ Recovery (\%) } & \multicolumn{2}{c}{ R.S.D. (\%) } \\
\hline Sample & \multicolumn{2}{c}{ Spiked } & \multicolumn{1}{c}{ (\% $(\mathrm{n}=5)}$. \\
Urine & Droxidopa & Carbidopa & Droxidopa & Carbidopa & Droxidopa & Carbidopa & Droxidopa & Carbidopa \\
& 0 & 0 & 0 & - & - & - & - & - \\
& 5.0 & 7.5 & 4.9 & 7.6 & 98.0 & 101.3 & 2.4 & 3.4 \\
& 10.0 & 12.5 & 10.2 & 12.3 & 102.0 & 98.4 & 3.2 & 1.8 \\
Tap & 15.0 & 17.5 & 14.6 & 17.9 & 97.3 & 102.3 & 1.9 & 2.4 \\
Water & 20.0 & 22.5 & 20.2 & 22.3 & 101.0 & 99.1 & 2.3 & 2.9 \\
& 0 & 0 & - & - & - & - & - & - \\
& 7.0 & 10.0 & 6.8 & 10.2 & 97.1 & 102.0 & 2.4 & 1.9 \\
& 12.0 & 12.5 & 12.4 & 12.3 & 103.3 & 98.4 & 3.3 & 2.4 \\
& 17.0 & 15.0 & 17.2 & 14.6 & 101.2 & 97.3 & 1.7 & 3.5 \\
& 22.0 & 17.5 & 21.9 & 17.7 & 99.5 & 101.1 & 2.3 & 2.6 \\
\hline
\end{tabular}

\section{Conclusion}

The construction of a chemically modified carbon paste electrode by the incorporation of $\mathrm{CuO}$ nanoparticles, ionic liquid and $\mathrm{Mn}$ (III) salen as modifying species is reported in this work. Excellent features, like a wide linear range and low detection limit proved the successful application of this sensor for the determinations of droxidopa. Moreover, the modified electrode presented wide linear range and high stability for the determination of droxidopa in the presence of carbidopa, suggesting this electrode as a good and attractive candidate for practical applications.

\section{Acknowledgements}

The authors acknowledge the financial support provided for this project (Project No. 98000318 and ethics code IR.KMU.REC.1398.264) by the Kerman University of Medical Sciences, Kerman, Iran.

\section{References}

[1] D.S. Goldstein, Cardiovasc. Drug Rev., 2006, 24, 189-203.

[2] H. Kaufmann, Clin. Auton. Res., 2008, 18, 19-24.
[3] C.J. Mathias, Clin. Auton. Res., 2008, 18, 25-29.

[4] S. Saito, K. Shioda, K. Nishijima, $J$. Clin. Psychopharma., 2012, 32, 428429.

[5] S. Perez-Lloret, M.V. Rey, A. PavyLe Traon, O. Rascol, Expert Opin. Orphan Drugs, 2014, 2, 509-522.

[6] K.V. Özdokur, E. Engin, Ç. Yengin, H. Ertaş, F.N. Ertaş, Anal. Lett., 2018, 51,73-82.

[7] R.A. Hauser, S. Isaacson, J.P. Lisk, L.A. Hewitt, G. Rowse, Mov. Disord., 2015, 30, 646-654.

[8] H.H. Takeda, T.A. Silva, B.C. Janegitz, F.C. Vicentini, L. H.C. Mattoso, O. Fatibello-Filho, Anal. Methods, 2016, 8, 1274-1280.

[9] C. Zapata-Urzúa, M. Pérez-Ortiz, M. Bravo, A.C. Olivieri, A. Álvarez-Lueje, Talanta, 2010, 82, 962-968.

[10] Q. Wang, M.R. Das, M. Li, R. Boukherroub, S. Szunerits, Bioelectrochem, 2013, 93, 15-22.

[11] D. Kul, C. Brett, Int. J. Electrochem., 2011, 2011.

[12] S.S. Badawy,Y.M. Issa, A.S. TagEldin, Electroanalysis, 1996, 8, 10601064. 
[13] Y. Li, X. Zhai, X. Liu, L. Wang, H. Liu, H. Wang, Talanta, 2016, 148, 362369.

[14] S. Esfandiari-Baghbamidi, $\mathrm{H}$. Beitollahi, S. Tajik, Ionics, 2015, 21, 2363-2370.

[15] D. Huang, H. Wu, Y. Zhu, H. Su, H. Zhang, L. Sheng, C. Song, Int. J. Electrochem. Sci., 2019, 14, 3792-3804. [16] S. He, P. He, X. Zhang, X. Zhang, K. Liu, L. Jia, F. Dong, Anal. chim. Acta, 2018, 1031, 75-82.

[17] A. Abellán-Llobregat, L. Vidal, R. Rodríguez-Amaro, A. Canals, E. Morallon, Electrochim. Acta, 2018, 285, 284-291.

[18] H. Beitollahi, F. Garkani-Nejad, S. Tajik, M.R. Ganjali, Iran. J. Pharmaceut. Res., 2019, 18, 80-90.

[19] N.S. Anuar, W.J. Basirun, M. Ladan, M. Shalauddin, M.S. Mehmood, Sens. Actuators B Chem., 2018, 266, 375-383.

[20] S. Chaiyo, E. Mehmeti, K. Žagar, W. Siangproh, O. Chailapakul, K. Kalcher, Anal. Chim. Acta, 2016, 918, 26-34.

[21] S. Esfandiari-Baghbamidi, $\mathrm{H}$. Beitollahi, S. Tajik, Anal. Bioanal. Electrochem., 2014, 6, 634-645.

[22] S. Sharma, N. Singh, V. Tomar, R. Chandra, Biosens. Bioelectron., 2018, 107, 76-93.

[23] M.M. Foroughi, H. Beitollahi, S. Tajik, A. Akbari, R. Hosseinzadeh, Int. J. Electrochem. Sci., 2014, 9, 8407.

[24] Q. He, J. Liu, X. Liu, Y. Xia, G. Li, P. Deng, D. Chen, Molecules, 2018, 23, 2130.

[25] H. Beitollahi, H. Karimi-Maleh, H. Khabazzadeh, Anal. Chem., 2008, 80, 9848-9851.

[26] Q. He, J. Liu, X. Liu, G. Li, D. Chen, P. Deng, J. Liang, Nanomaterials, 2018, 8, 194.

[27] H. Beitollahi, S. Tajik, R. Alizadeh, J. Electrochem. Sci. Technol., 2017, 8, 307-313.
[28] H. Karimi-Maleh, C.T. Fakude, N. Mabuba, G.M. Peleyeju, O.A. Arotiba, J. Colloid Interf. Sci., 2019, 554, 603610.

[29] F. Tahernejad-Javazmi, M. Shabani-Nooshabadi, H. Karimi-Maleh, Composites Part B, 2019, 172, 666-670. [30] M.R. Ganjali, Z. Dourandish, H. Beitollahi, S. Tajik, L. Hajiaghababaei, B. Larijani, Int. J. Electrochem. Sci., 2018, 13, 2448-2461.

[31] A. Khodadadi, E. Faghih-Mirzaei, H. Karimi-Maleh, A. Abbaspourrad, S. Agarwal, V.K. Gupta, Sens. Actuators B: Chem., 2019, 284, 568-574.

[32] M. Miraki, H. Karimi-Maleh, M. A.Taher, S. Cheraghi, F. Karimi, S. Agarwal, V. K. Gupta, J. Mol. Liq., 2019, 278, 672-676.

[33] H. Soltani, H. Beitollahi,A. H. Hatefi-Mehrjardi, S. Tajik, M. Torkzadeh-Mahani, Anal. Bioanal. Electrochem., 2014, 6, 67-79.

[34] H. Karimi-Maleh, M. Sheikhshoaie, I. Sheikhshoaie, M. Ranjbar, J. Alizadeh, N. Wendy Maxakato, A. Abbaspourrad, New J. Chem., 2019, 43, 2362-2367.

[35] D. Sangamithirai, S. Munusamy, V. Narayanan, A. Stephen, Mater. Sci. Eng. $C, \mathbf{2 0 1 8}, 91,512-523$.

[36] S.D. Bukkitgar, N.P. Shetti, Mater. Sci. Eng. C, 2016, 65, 262-268.

[37] M.A. El Mhammedi, M. Bakasse, R. Bachirat, A. Chtaini, Food Chem., 2008, 110, 1001-1006.

[38] H. Beitollahi, S. Ghofrani-Ivari, M. Torkzadeh-Mahani, Biosens. Bioelectron., 2018, 110, 97-102.

[39] Z. Liang, H. Zhai, Z. Chen, S. Wang, H. Wang, S. Wang, Sens. Actuators B Chem., 2017, 244, 897-906. [40] P.K. Kalambate, C.R. Rawool, S.P. Karna, A.K. Srivastava, Mater. Sci. Eng. $C$, 2016, 69, 453-461.

[41] H. Mahmoudi Moghaddam, H. Beitollahi, S. Tajik, S. Jahani, H. Khabazzadeh, R. Alizadeh, Russ. J. Electrochem., 2017, 53, 452-460. 
[42] N.G. Mphuthi, A.S. Adekunle, O.E. Fayemi, L.O. Olasunkanmi, E.E. Ebenso, Sci. Rep., 2017, 7, 43181.

[43] M.M. Rahman, M.M. Alam, A.M. Asiri, M.A. Islam, $R S C A d v ., 2017,7$, 22627-22639.

[44] H. Beitollahi, S. Tajik, Environ. Monit. Assess., 2015, 187, 257.

[45] X. Li, H. Wen, Q. Fu, D. Peng, J. Yu, Q. Zhang,X. Huang, App. Surf. Sci., 2016, 363, 7-12.

[46] L.A. Alshahrani, L. Liu, P. Sathishkumar, J. Nan, F.L. Gu, J. Electroanal. Chem., 2018, 815, 68-75.
[47] H. Huang, Y. Liu, J. Wang, M. Gao, X. Peng, Z. Ye, Nanoscale, 2013, 5, $1785-1788$.

[48] Z. Guo, M.L. Seol, M.S. Kim, J.H. Ahn, Y.K. Choi, J.H. Liu, X.J. Huang, Nanoscale, 2012, 4, 7525-7531.

[49] J.S. Shaikh, R.C. Pawar, A.V. Moholkar, J.H. Kim, P.S. Patil, Appl. Surf. Sci., 2011, 257, 4389-4397.

[50] H. Karimi-Maleh, M. Sheikhshoaie, I. Sheikhshoaie, M. Ranjbar, J. Alizadeh, N.W. Maxakato, A. Abbaspourrad, New J. Chem., 2019, 43, 2362-2367.

[51] H. Beitollahi, I. Sheikhshoaie, Int. J. Electrochem. Sci., 2012, 7, 7684-7698.

How to cite this manuscript: Mohammad Reza Aflatoonian, Somayeh Tajik, Behnaz Aflatoonian, Iran Sheikh Shoaie, Mahdieh Sheikhshoaie, Hadi Beitollahi Copper oxide, ionic liquid and Mn(III) Salen modified carbon paste electrode as selective electrochemical sensor for determination of droxidopa in the presence of carbidopa. Eurasian Chemical Communications, 2020, 2(3), 387-397. 